\title{
The locus coeruleus and cognitive function: Attempts to relate noradrenergic enhancement of signal/noise in the brain to behavior
}

\author{
SUSAN J. SARA \\ Centre National de la Recherche Scientifique, Gif-sur-Yvette, France
}

\begin{abstract}
Behavioral manipulations that alleviate forgetting in a complex maze task are paralleled by pharmacological manipulations which modulate noradrenergic activity. Increased noradrenergic activity is observed following prior exposure to contextual reminder cues. It is proposed that the facilitated retrieval reflects a conditioned arousal and an enhanced attention to discriminative stimuli mediated by the noradrenergic response. Failure to find learning and memory retrieval deficits in rats that are depleted of noradrenaline is discussed in terms of functional recovery after lesions.
\end{abstract}

That the locus coeruleus (LC)-forebrain noradrenergic projection should play an important role in the control of attention and integrative activity involved in cognitive functions is a view held by many investigators (see, e.g., Aston-Jones, 1985; Hobson, 1980; Moore, 1980; Segal, 1985). This hypothesis is based on the electrophysiological studies of neuromodulatory properties of noradrenaline (NE) and the action of NE in the control of signal/noise in the forebrain. In spite of the enthusiasm for such a hypothesis of LC function, there remains a very large gap between the electrophysiological observations and supporting behavioral evidence that this modulation is related to attention or other cognitive functions. In fact, the lack of robust behavioral effects of lesion to the NE system has led several authors to question whether this system is, indeed, involved in attention, learning, or memory processes at all (Crow, Deakin, File, Longden, \& Wendlandt, 1978; Pisa \& Fibiger, 1983; Robbins \& Everitt, 1985).

The purpose of this paper is threefold: (1) to consider some reasons for the lack of clear-cut behavioral evidence to support this intuitively attractive hypothesis; (2) to present some new behavioral data which do lend some support; and (3) to discuss alternative behavioral approaches which might be useful in bridging the gap be-

\footnotetext{
This article is based on a roundtable discussion held at the European Brain and Behavior Society Workshop on Brain Plasticity, Learning and Memory held August 30-September 1, 1984, in Strasbourg, France.

The experiments reported here were carried out with the technical assistance of Michèle Dumas. Neurochemical analysis was performed by Bernard Guibert and Vincent Leviel. The research was funded by a grant from the Ministry of Industry and Research of France, ATP 82 F 0689. The author thanks Serge Laroche, William McEntee, and Menahem Segal for helpful comments on an earlier version of this manuscript. The author's mailing address is: Laboratoire de Physiologie Nerveuse, Département de Psychophysiologie, F91190 Gif-sur-Yvette, France.
}

tween electrophysiological evidence, on the one hand, and cognitive processes, on the other.

\section{ANATOMICAL AND ELECTROPHYSIOLOGICAL BACKGROUND}

The "new look at the aminergic nervous system" proposed by Dismukes (1977) was based on the report that most of the variscosities of noradrenergic or serotonergic neurons have no direct postsynaptic elements. Thus, when the neurotransmitter is released, it does not bind immediately but diffuses into the intercellular space to affect neurons at great distances from the site of release (Descarries, Watkins, \& Lapierre, 1977). This particular anatomical arrangement suggested to many investigators that such neurons might be more involved in modulation of the reactivity of other neurons rather than in transmission of specific information. More recent anatomical studies, however, show a laminar organization of NE fibers and a much higher number of direct postsynaptic receptor sites than described by earlier studies (Olschowka, Molliver, Grzanna, Rice, \& Coyle, 1981).

Electrophysiological studies have, nevertheless, confirmed the function of $\mathrm{NE}$ as a neural modulator. In the presence of iontophoretically applied NE, excitatory responses to acetylcholine and inhibitory responses to GABA are both enhanced (e.g., Woodward, Moises, Waterhouse, Hoffer, \& Friedman, 1979).

$\mathrm{NE}$ in the hippocampus, released by electrical stimulation of the LC, can enhance neuronal responses to stimuli that predict reinforcement, while inhibiting, at the same time, spontaneous background activity (Segal \& Bloom, 1976). This increased signal/noise, as these authors suggested, might result in improved selective attention. It was later shown, in the cat cortex, that neuronal responses evoked by visual stimuli were enhanced in the presence of NE, especially responses of neurons receiving stimuli 
in their preferred direction or orientation. At the same time, background activity was suppressed, thus improving signal/noise (Madar \& Segal, 1980). In this case, it should result in enhanced visual acuity and facilitated behavioral-visual function (Segal, 1985).

Responses to somatosensory stimuli can be enhanced when NE is applied to the cortex (Waterhouse, Moises, \& Woodward, 1980). These authors, too, suggest that this enhancement of the signal by NE might be a mechanism of regulation of selective attention. A similar role for NE in the olfactory bulb (Gervais \& Pager, 1983) and the cochlear nucleus (Pickles, 1976) has been suggested.

\section{BEHAVIORAL TESTS OF THE ATTENTION HYPOTHESIS}

\section{Lesion Studies}

Functional hypotheses can be tested by enhancing or impairing the activity of a given structure or system, either chronically through electrolytic or neurotoxic lesions, or reversibly with pharmacological treatments or intracranial electrical stimulation concurrent with behavioral analysis. In dealing with the LC system, by far the preferred method has been preparing rats with neurotoxic lesions to the ascending noradrenergic bundle. And, indeed, some of the earliest support for the selective attention hypothesis came from behavioral studies following lesions of this type (Mason \& Iversen, 1978). These authors and others (e.g., Lorden, Rickert, Dawson, \& Pellymounter, 1980) showed an absence of latent inhibition and an increased resistance to extinction in such rats, results which they interpreted as indicative of an inability to ignore irrelevant stimuli, that is, a deficit in selective attention. More recent studies have, however, either failed to confirm earlier findings (e.g., Pisa \& Fibiger, 1983) or rejected interpretations of the behavioral deficits in terms of failure to screen out irrelevant stimuli. In fact, recent reports from two laboratories suggest that NE-depleted rats integrate less information concerning context, indicating a narrowed scope of stimulus selection, rather than a broadened one, as proposed earlier by Mason and Iversen (see Archer, Mohammed, \& Jarbe, 1983; Robbins \& Everitt, 1985).

It is not our aim to review this vast literature concerning behavioral effects of lesion to the LC-dorsal bundle system. This has been done many times, most recently and thoughtfully by Robbins and colleagues (Robbins, 1984; Robbins \& Everitt, 1985), whose latest conclusion is that behavioral studies have not produced strong support for the notion that LC-forebrain projection is involved in selective attention processes, although they suggest that the tests used to measure attention may not be adequate. The question of the validity of the behavioral measure is of utmost importance in evaluating the role of NE in attention and other cognitive processes, and it is certainly questionable whether latent inhibition, resistance to extinction, and other paradigms that have been used in these studies are, indeed, valid. On the other hand, the question of persistence of the functional effects of the lesion has not been adequately addressed. Although long-lasting effects on taste neophobia are cited as evidence for lack of functional recovery after NE lesion (Robbins \& Everitt, 1985), we still feel that there is a very distinct possibility that such recovery accounts for the absence of robust and reliable cognitive deficits in the literature.

Functional recovery after NE depletion. Although it is well established that NE can remain depleted for a long time after neurotoxic lesions, there is marked and important recovery of functions dependent upon NE in spite of a persistence of the depletion weeks after the lesion. For example, after depletion of cortical $\mathrm{NE}$ by lesion to the LC, there is a marked change in oxidative metabolism (LaManna, Harik, Light, \& Rosenthal, 1981) and in cyclic AMP production. These processes gradually return to normal, 2-8 weeks after lesion, even while forebrain NE remains depleted (Harik et al., 1981).

Na K-ATPase-the electrogenic pump-is thought to be activated by NE (Segal, 1981; Segal, Sagie, \& Mayevsky, 1980), and the activity of this enzyme is markedly reduced following neurotoxic lesions to the NE system. Again, the activity returns to normal within 8 weeks in the face of continued NE depletion (Swann, 1984). The mechanism of recovery is not known, although it is possible that dopamine (DA) and/or serotonin (5HT) take over function of NE, at least in some brain regions. In fact, many neurons in the rat frontoparietal cortex have been found to be inhibited by all three amines (Reader, 1983). Some support for this notion comes from a recent study by Harik (1984), who found that cortical DA activity is enhanced after depletion of NE. He suggests that this may underlie the functional recovery which he and his collaborators have seen in the face of persistent NE depletion (Harik, 1984).

There have been few studies of electrophysiological effects of lesion to the coeruleocortical system, but the question of functional recovery might be studied by examining the postlesion time course of any such effects. It is possible that after loss of NE, its signal-to-noise enhancing properties in target cortical areas are accomplished by another mechanism. Indeed, there is evidence that $5 \mathrm{HT}$ has a modulatory action similar to that of NE in the hippocampus in that it inhibits spontaneous activity while enhancing activity evoked by stimulation of the commissural path (Segal, 1982). Moreover, as noted above, many cortical cells can be inhibited by DA, NE, and 5HT.

Still another mechanism of recovery after NE depletion might involve changes in the NE neurons themselves. There are important modifications in sensitivity of these neurons, both pre- and postsynaptically, after partial destruction to the system. Their inhibitory autoreceptors become less sensitive to the effects of NE or receptor agonists, resulting in an increase of firing in the spared LC neurons (Chiodo, Acheson, Zigmond, \& Stricker, 1983). Moreover, there is a marked increase $(70 \%)$ in postsynaptic beta receptor binding in cortex, with no changes in alpha receptors, two weeks after lesion of the LC (Harik et al., 1981). 
This recovery then could account for the lack of dramatic effects of NE lesions on behavior presumed to measure cognitive function in the rat. The functional recovery begins as early as 2 weeks after the lesion, and this is well within the recovery and testing period of most studies of behavioral effects of lesions. Again, it is important to emphasize that this functional recovery occurs even when NE remains depleted. Most investigators control for the effectiveness and durability of lesions by measuring forebrain NE after the behavioral experiments. Whatever the mechanism, such recoveries underline the compensatory capacity of the cerebral cortex and the importance of considering time-dependent recovery of function in the face of persistent NE depletion when assessing effects of lesions to this system.

NE and developmental plasticity. A similar discussion has arisen concerning research on the role of NE in developmental cortical plasticity. In a series of convincing experiments, it was shown that a normally functioning NE system in the visual cortex is needed for maintaining visuocortical plasticity in the neonatal kitten (e.g., Kasamatsu \& Pettigrew, 1976; Kasamatsu, Pettigrew, \& Ary, 1979). These results were, however, recently challenged by several groups who failed to find any deficit in plasticity after lesions to the NE system (Adrien et al., 1982; Daw, Rader, Robertson, \& Videen, 1983). An important, and perhaps crucial, difference between Kasamatsu's approach and that of other laboratories is that he blocks or stimulates NE activity locally, with assays of plasticity carried out as soon as possible after the treatment, whereas others have used general and chronic NE depletion, with the assays carried out long after the neurotoxic intervention, that is, after ample time for functional recovery (see Kasamatsu \& Shirokawa, 1985). Interestingly, activation of the beta receptor is crucial for this plasticity (Kasamatsu \& Shirokawa, 1985). As noted above, it is the beta receptor which shows a supersensitivity, 2 weeks after the lesion, so functional recovery must be a major consideration when interpreting these results. The problem is similar to that of trying to demonstrate a role for NE in behavioral plasticity and selective attention.

NE depletion after treatment with DSP4. DSP4 is a neurotoxin that is selective for NE; injected systemically, there is a total recovery of peripheral NE within 1 week and a partial recovery $(+50 \%)$ of hypothalamic and cerebellar NE; cortical structures and spinal cord NE have been reported to remain depleted for weeks or months after treatment (Hallman, Sundström, \& Jonsson, 1984). Forebrain NE depletion has been reported to be correlated with an absence of postdecapitation clonus (Archer, Cotic, \& Järbe, 1982), and we have confirmed this in our laboratory (Guibert, Sara, \& Leviel, 1983).

Rats were injected with DSP4 (50 mg/kg, ip) or an equal volume of saline ( $n=8$ in each group). Ten days later, the rats were decapitated, the postdecapitation clonic reflex (PDR) latency was noted, and the brains were dissected into cortex, hippocampus, hypothalamus, striatum, and brainstem. Catecholamines were measured by highpressure liquid chromatography (HPLC) with electrochemical detection.

All of the control animals had a PDR latency of less than $2 \mathrm{sec}$ and all DSP4-treated rats had a latency of more than $20 \mathrm{sec}$; most showed a total absence of PDR. The results of the neurochemical analysis can be seen in Tables 1 and 2. Hypothalamic and brainstem NE levels showed considerable recovery, whereas cortex and hippocampus were depleted to nondetectable levels in all eight treated rats. There was no effect on dopamine or serotonin or their metabolites, as measured in the striatum. These results are very similar to those reported by other groups (e.g., Ross, 1976).

Learning and memory after NE depletion by DSP4. In a subsequent behavioral experiment, rats were treated with DSP4 (50 mg/kg ip), and when they had recovered their body weight they were trained in the same appetitive maze task used in the pharmacological studies described in the next section. Three weeks after training, they were tested for retention under the same conditions as used in training, and were then submitted to four daily reversal trials. At the completion of behavioral testing, 14 weeks after the DSP4 treatment, the latency and duration of PDR were noted.

There were two distinct populations of DSP-treated rats-those that had an absence of PDR or a PDR with a latency $>20 \mathrm{sec}$ and those that had nearly normal PDR, that is, a latency $<2 \mathrm{sec}$ and a duration of $+20 \mathrm{sec}$. Subsequent HPLC analysis of a randomly selected sample of the group $(n=14)$ showed that those rats without PDR had undetectable levels of hippocampal NE (with one exception), whereas those with normal PDR had normal or above normal levels of NE (with one exception; see Figure 1).

For statistical analysis, the behavioral data of the DSP4treated rats were divided into two groups: those with normal PDR $(n=14)$ and those showing no PDR $(n=14)$. The number of errors at each trial of acquisition can be seen in Figure 1. Analysis of variance showed a significant effect of treatment $[F(2,52)=4.62, p=.01]$, an effect of repetition $[F(6,32)=25.89, p<.001]$, and a sig-

Table 1

Noradrenaline Levels in Various Brain Regions 10 Days After DSP4 Treatment

\begin{tabular}{lcccc}
\hline & Cortex & Hippocampus & Hypothalamus & Brain Stem \\
\hline DSP4 & ND & ND & $10.01 \pm .53$ & $11.05 \pm .41$ \\
Control & $1.92 \pm .1$ & $2.06 \pm .26$ & $20.2 \pm 4.15$ & $4.3 \pm .7$ \\
\hline
\end{tabular}

Note-Results expressed in $\mathrm{ng} / \mathrm{mg}$ protein. $N D=$ nondetectable levels. Rats were injected with $50 \mathrm{mg} / \mathrm{kg}$ ip DSP4 or $2 \mathrm{ml}$ of saline.

Table 2

Striatal Dopamine, Serotonin, and Metabolites 10 Days After DSP4 Treatment

\begin{tabular}{lcccc}
\hline & DOPAMINE & DOPAC & 5-HT & 5-HIAA \\
\hline DSP4 & $150.33 \pm 6.9$ & $5.93 \pm .49$ & $4.21 \pm .5$ & $.34 \pm .04$ \\
Control & $151.88 \pm 8.3$ & $6.34 \pm .18$ & $4.34 \pm .52$ & $.37 \pm .02$ \\
\hline
\end{tabular}




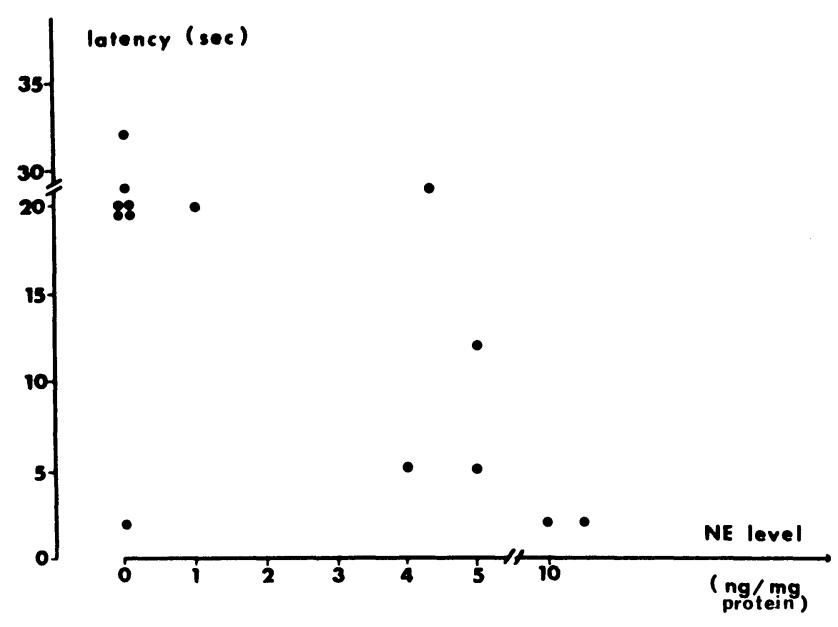

Figure 1. Postdecapitation latency for vigorous movement of limbs (postdecapitation reflex) as a function of hippocampal noradrenaline levels for rats treated with DSP4, 14 weeks previously. Note that, with one exception, rats with low or nondetectable levels of NE had latencies $>20 \mathrm{sec}$, whereas those with normal or higher than normal levels of NE had latencies of $<15$ sec. Untreated rats show this PDR within $5 \mathrm{sec}$ of decapitation (Guibert, Sara, \& Leviel, 1983).

nificant trials $\times$ treatments interaction $[\mathrm{F}(12,312)=$ $1.854, \mathrm{p}=.04]$. As can be seen in Figure 2, there is clearly no difference in the behavior, at this point, between the two subgroups of lesioned rats.

At the retention test, however, the DSP4-treated rats with no PDR and, presumably, little or no NE tended to make fewer errors than did saline controls (Figure 3 ). Although this difference is not significant for all three groups $[F(2,52)=1.99, p=.14]$, when the low NE group alone is compared with the control, the difference is significant $[F(1,39=3.71, p=.05]$. Note that the withingroup variance would likely have been less, and the between-group variance greater, if the post hoc group assignments could have been made on the basis of a precise neurochemical analysis, rather than on the basis of an implicit measure of NE, the PDR.

A selection of rats that exhibited good performance at the retention test was retained for the reversal study. They were submitted to several retraining trials, such that there was no difference between the performance of the lesioned and control rats before reversal training. The number of errors during reversal is shown in Figure 4; the low-NE rats acquired the reversal faster than control animals [interaction significant, $\mathrm{F}(3,51)=3.47, \mathrm{p}=.02]$.

There are several important issues raised by these results. This is certainly not the first time that a NE depletion has failed to impair cognitive function. In fact, most of the literature is in agreement on this point. On the other hand, the facilitation of retrieval and reversal in those rats showing no recovery of NE is surprising, although a similar result has recently been reported: reversal of a spatial task was facilitated after 6OHDA lesions to the septum (which depletes about $30 \%$ of hippocampal NE) (Harrell, Barlow, Miller, Haring, \& Davis, 1984). As in our experiments, the reversal aspect of the task is necessarily performed long after the lesion and extensive training,

\section{ACQUISITION}

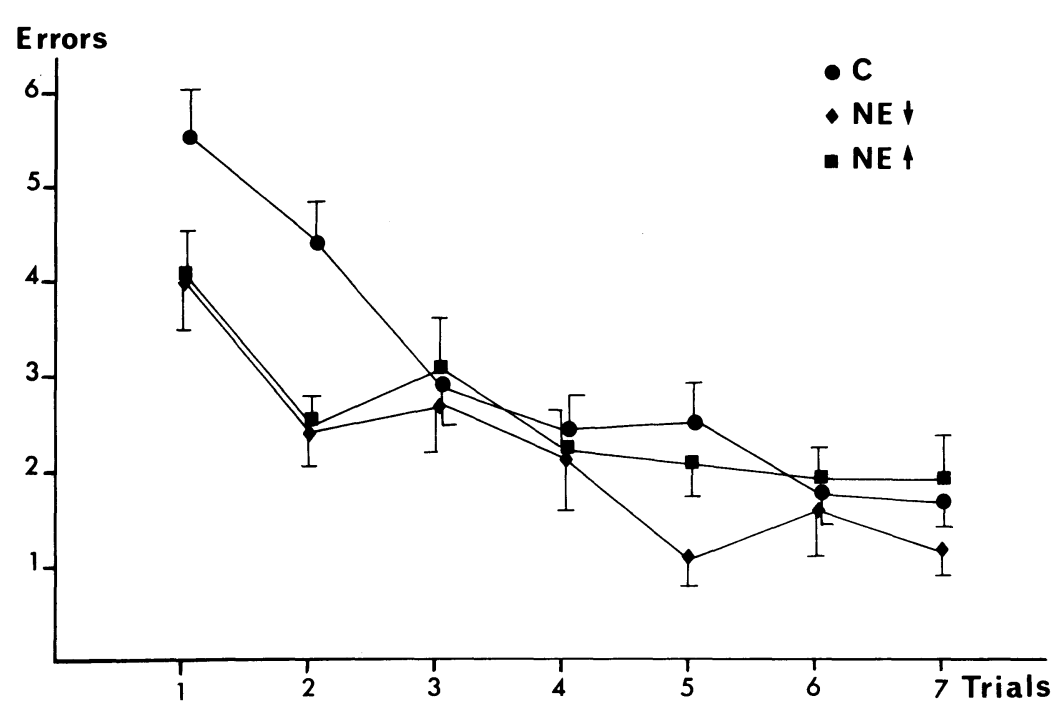

Figure 2. Acquisition of the six-unit linear maze over 7 days. $\mathrm{C}=$ control group; $\mathrm{NE} \uparrow=$ DSP4-treated rats with normal postdecapitation reflexes (PDR) and, implicitly, high NE when sacrificed; NE $\downarrow$ = DSP4-treated rats with no PDR or a latency $>20$ sec and, implicitly, no NE when sacrificed. Note that there is no difference between the two DSP4-treated groups. The significant interaction indicates that at the first two trials fewer errors were made by the two DSP4 groups than by the control group. 


\section{RETRIEVAL}

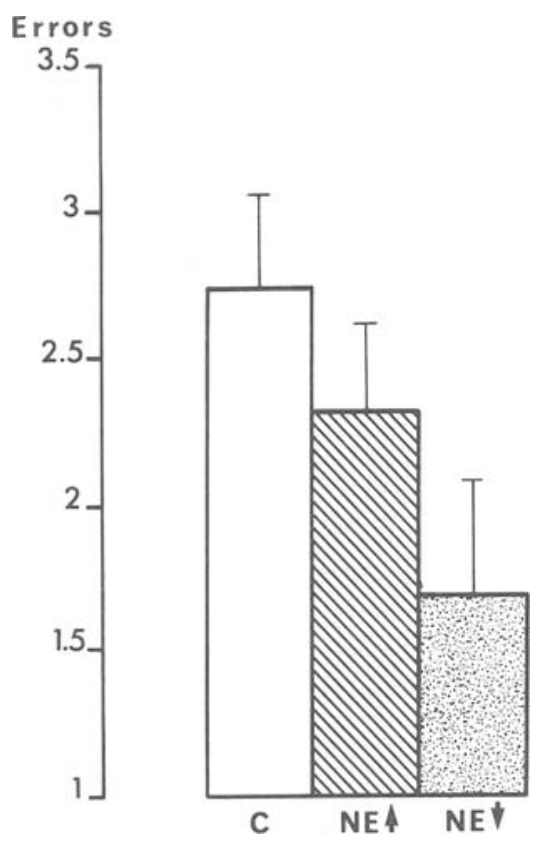

Figure 3. Number of errors at the retention test, 25 days after the last acquisition trial.

allowing ample time for recovery of function, independent of recovery of NE. In the case of a partial denervation, increase in firing of the remaining cells might be expected (Chiodo et al., 1983), which could account for the improved performance of the lesioned group, especially if there is an overcompensation or receptor supersensitivity.

Another puzzling point is the recovery of NE in some DSP4-treated animals but not in others. As indicated above, most laboratories have found persistent depletion of NE levels for months after treatment with DSP4. Our own preliminary experiments showed depletion in $100 \%$ of rats, 10 days after treatment (Table 1 ), which strongly suggests that the NE seen in half of the treated rats in the behavioral experiment is due to recovery during the 14 weeks of behavioral studies, rather than to ineffective DSP4 treatment. This recovery could be due to a number of factors, such as an increase in firing of neurons in the LC or sprouting of new axonal terminals. Intensive manipulation of the animals, along with the stress of food deprivation during the learning experiments, might induce or accelerate such regenerative processes. We do not know why this should occur in some experimental animals and not others, but a more thorough study of the interaction between environmental variables, stress, and nutrition, on the one hand, and recovery after NE lesions, on the other, is certainly warranted.

\section{Behavioral Effects of Enhancement of LC Activity}

Acute, reversible modification of the LC system, electrically or pharmacologically, might be a more appropriate strategy to use in addressing the question of the behavioral significance of NE modulation of neuronal activity. There is, to our knowledge, only one study that has used direct stimulation of the LC in freely moving animals (Segal \& Edelson, 1978). Priming stimulation of the LC facilitated reversal of brightness discrimination, but the effect was seen only on latency measures. We are

\section{REVERSAL}

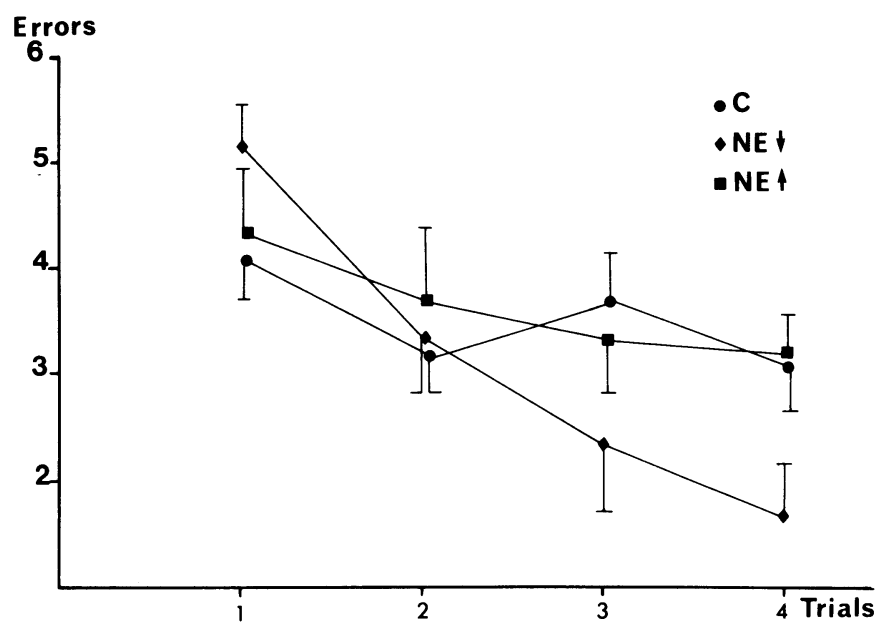

Figure 4. Acquisition of a reversal of the linear maze. The animals were tested 25 days after training (Figure 2), and then retrained. The rats with the best retraining performance were retained for the reversal experiment. The significant interaction reflects the better performance of the NE $\downarrow$ rats at the last two trials. Note that the performance of the control and the NE 1 rats after four reversal trials is still at chance level, whereas the NE $\$ rats show a significant decrease in number of errors across trials. 
planning further experiments of this type, using behavioral tasks such as complex discriminations and forgetting paradigms, which make attentional demands on the animal and are sensitive to pharmacological enhancement of NE. We have found that priming stimulation of the mesencephalic reticular formation (MRF) alleviates forgetting of a complex maze path after a long retention interval, if it is administered just before the retention test (Sara, Deweer, \& Hars, 1980). Very recent results from our laboratory have shown that this MRF stimulation increases NE in the hippocampus, but not in the hypothalamus, suggesting that fibers of the dorsal noradrenergic bundle, which pass close by the point of the electrode placement, might be stimulated, resulting in release of NE in the forebrain.

There are relatively few pharmacological studies designed to directly evaluate the role of NE in selective attention. This is surprising, since there is a large pharmacopeia of rather selectively acting agents with which to manipulate this system. There is, however, a substantial literature that provides some support for the hypothesis, first put forth by Kety (1970), concerning the role of NE in memory processes. This pharmacological literature was critically reviewed recently by Quartermain (1983), who concluded that "while most of the individual studies do not provide unambiguous support for adrenergic involvement in memory, the collective evidence favors a role of NE' (p. 44). Some of the ambiguity in the results might be due to differences in the degree of selective attention required by the different paradigms used in these memory studies. For example, less reliance on selective attention would be required in the acquisition and retrieval of simple tasks with salient discriminanda, presented within a well-defined context, than in complex tasks using more subtle stimuli. In the latter case, the organism is required to selectively attend to the significant aspects of the stimuli, while inhibiting responding to the irrelevant. Noradrenergic involvement should be more readily demonstrable in such situations.

Contextual cue reminders and LC activity: A hypothesis. For the past several years, we have been using an appetitive maze task to study long-term retention and retrieval processes in the rat (Deweer \& Sara, 1984; Deweer, Sara, \& Hars, 1980; Sara, Deweer, \& Hars, 1976). This task was originally assumed to be a straightforward spatial discrimination task (although its retrieval after a long retention interval is highly dependent upon context; see Sara, 1985). A recent experiment, however, suggests that rats rely to a large extent on very subtle intramaze cues in the form of complex configurations of shadows on the floor and walls of the maze. This discovery was serendipitous, in that it occurred after the physical location of the maze in the laboratory was changed; the lighting in the new location was much more diffuse, providing no intramaze shadows. Under the new conditions, the rats failed to learn at the usual rate, but once the former lighting conditions were restored, the rats learned at the rate observed in the previous experiments (Sara \& Dekeyne, 1985).
The maze consists of six successive left-right choices, the correct response being LRRLLR, or its mirror image. The rat is rewarded with 2 min access to food pellets in the goalbox. With one trial per day, a rat usually learns this task in 5-7 trials. Tested 3-4 weeks later, there is significant forgetting, as measured by run time and by errors, the performance being at about the same level as on the third day of acquisition. (A full description of the apparatus and procedure can be found in Deweer et al., 1980.) When the rat is exposed to the context in which it was trained, for $30-90 \mathrm{sec}$ before the test, there is a marked alleviation of the forgetting; context-reminded rats make fewer errors than those submitted to various "pseudo-reminder" procedures (Deweer \& Sara, 1984; Deweer et al., 1980).

These experiments, demonstrating the modulation of the retrieval of weak memories by context or background, have led us to propose that contextual cue reminders elicit a preparatory conditioned response, which, in turn, facilitates responding to the nominal conditioned stimulus (CS). Such a preparatory conditioned response to context was already implicit in Pavlov's work. Kupalov (1961) gave it the name "truncated conditioned reflex." The context, or experimental room, because of its association with the reinforcement, comes to elicit a conditioned tonic arousal response which puts the organism in a state for efficient responding to the explicit CS. This preparatory response is highly resistant to extinction (see Konorski, 1967). A good example of this durable conditioned response is the emotional response we have all experienced to a place in which a significant event occurred in the distant past. Often we are unable to immediately recall the explicit event that occurred within this context, but the preparatory response to this context persists. It would seem to be highly resistant to forgetting, as well as especially resistant to extinction. Experimental evidence for this particularly long retention of the significance of the context is provided by a recent experiment showing that in rats, the efficacy of various cuing procedures changes with the time between acquisition and retrieval. After long retention intervals, cues of a contextual nature are more effective than the nominal CS or the unconditioned stimulus in reinstating memory, suggesting that the significance of the nominal stimulus is forgotten at a faster rate than the significance of the context (Gisquet-Verrier, Dekeyne, \& Alexinsky, 1985).

To carry this line of thinking a step further in our attempt to understand how contextual cue reminders might facilitate retrieval of weak memories, we have suggested that conditioned firing of LC neurons in response to context might be part of this preparatory conditioned reflex (Sara, 1985). The resultant release of NE in such target structures as sensory cortex or hippocampus would enhance signal/noise and thereby facilitate selective attention, as suggested by several authors and discussed above.

Facilitation of memory retrieval and discrimination learning by yohimbine. If the contextual cue reminder facilitates performance after a long retention interval by eliciting an LC response, then similar behavioral facili- 
tation should be obtained by stimulating this system pharmacologically. Amphetamine, which releases both NE and DA, facilitated retrieval in this maze-forgetting paradigm when it was injected before the retention test. There was no effect of this drug when it was injected at any time during acquisition (Sara \& Deweer, 1982).

Recent studies, using the same paradigm, have shown that yohimbine, an NE alpha 2 receptor antagonist, is also effective in alleviating forgetting. At the active doses of 0.5 or $1.0 \mathrm{mg} / \mathrm{kg}$, yohimbine is fairly specific to the alpha 2 receptor and increases firing of LC cells by blocking presynaptic inhibition (Goldberg \& Robertson, 1983). The result is an increase in release of NE in target forebrain structures. The doses used did not produce any effects on spontaneous behavior such as hole board exploration or locomotor activity (Sara \& LeRoch, 1985).

Sixty rats were trained for seven daily trials in the linear maze. Forty-five rats that had made fewer than three errors at the last training trial were retained for testing. The acquisition curve for time is shown on the left in Figure 5; the curve for errors is shown in Figure 6. The animals were divided into matched groups according to training performance. Twenty-five days after the last training trials, they were tested for retention. Twenty-five minutes before the retention test, they were injected with saline $(1 \mathrm{mg} / \mathrm{kg}$ ip) or yohimbine $(0.5 \mathrm{or} 1.0 \mathrm{mg} / \mathrm{kg}$, yohimbine hydrochloride, Sigma).
There was no drug effect on time to run the maze, as can be seen in Figure 5. All rats took a longer time to run than they had at the last training trial. Saline-treated rats showed a robust forgetting in that they made more errors at retention test than at the last training trial. Drugtreated rats made no more errors at testing than they had on the last training trial-an alleviation of forgetting. The two doses of yohimbine were equally effective, as can be seen in Figure 6. A contrast analysis between combined drug groups and the saline control group was significant $[\mathrm{F}(1,44)=4.08, \mathrm{p}<.05]$.

It is unlikely that the improved maze performance in yohimbine-treated rats is due to nonspecific effects such as drug-induced changes in locomotor activity or motivation, since there were no differences in run time (Figure 5) and no differences in latencies or amount of food consumed in the goalbox.

A subsequent experiment verified that yohimbine has no nonspecific effect on performance in the maze. The original purpose of this experiment was to control for such possible performance effects and to see if the facilitation was limited to conditions under which control animals forget. A similar control experiment revealed that amphetamine, although facilitating memory retrieval after a long retention interval, had no effect whatsoever when administered at various stages of acquisition (Sara \& Deweer, 1982).

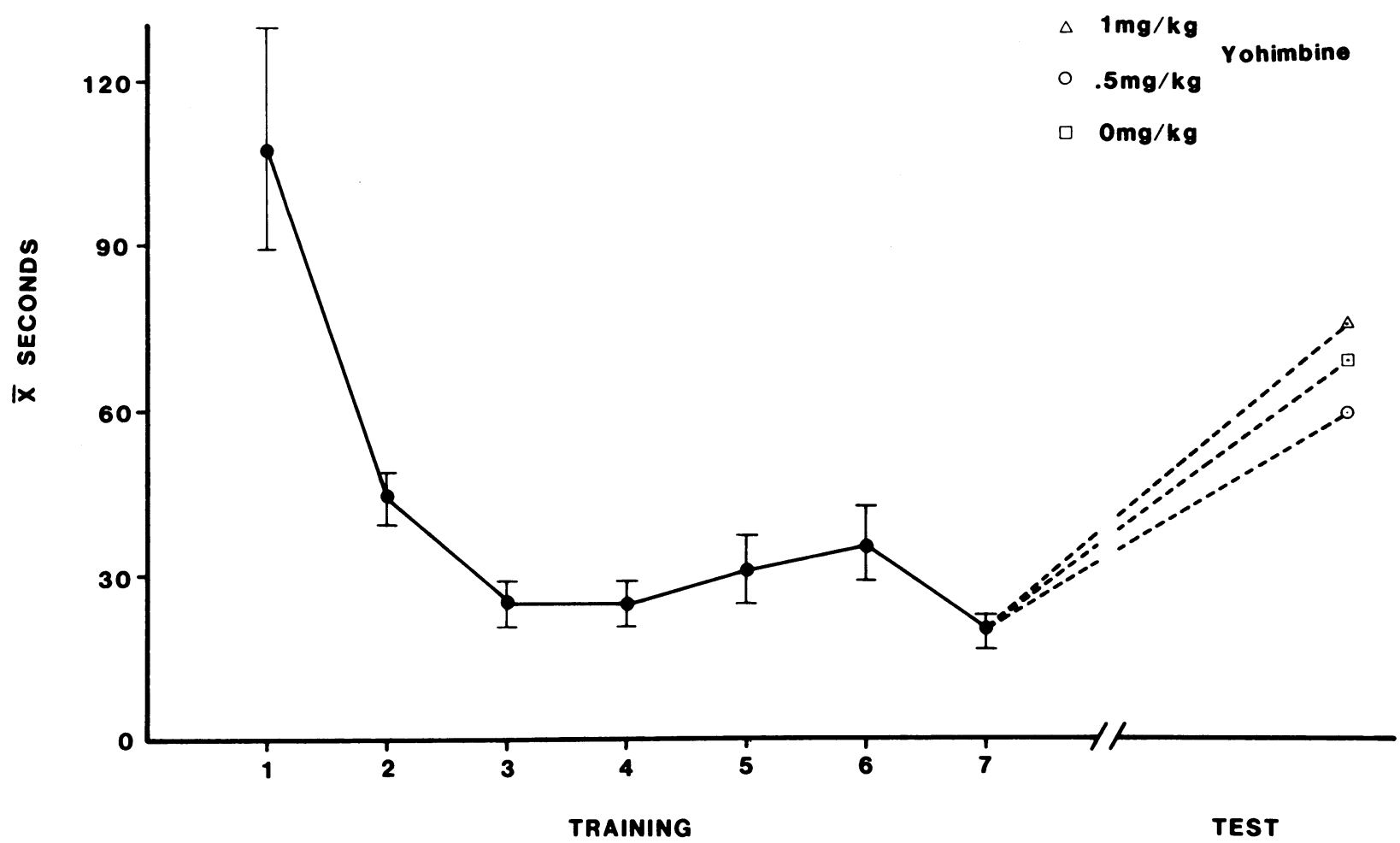

Figure 5. Time in seconds during acquisition of the linear maze task for the combined groups before treatment is seen at the left. Time to run the maze at retention test, $\mathbf{2 5}$ days after acquisition, for each treatment group is seen at the right of the figure. All animals take longer to run the maze at retention test than at the last training trials. There is no effect of yohimbine on run time. 


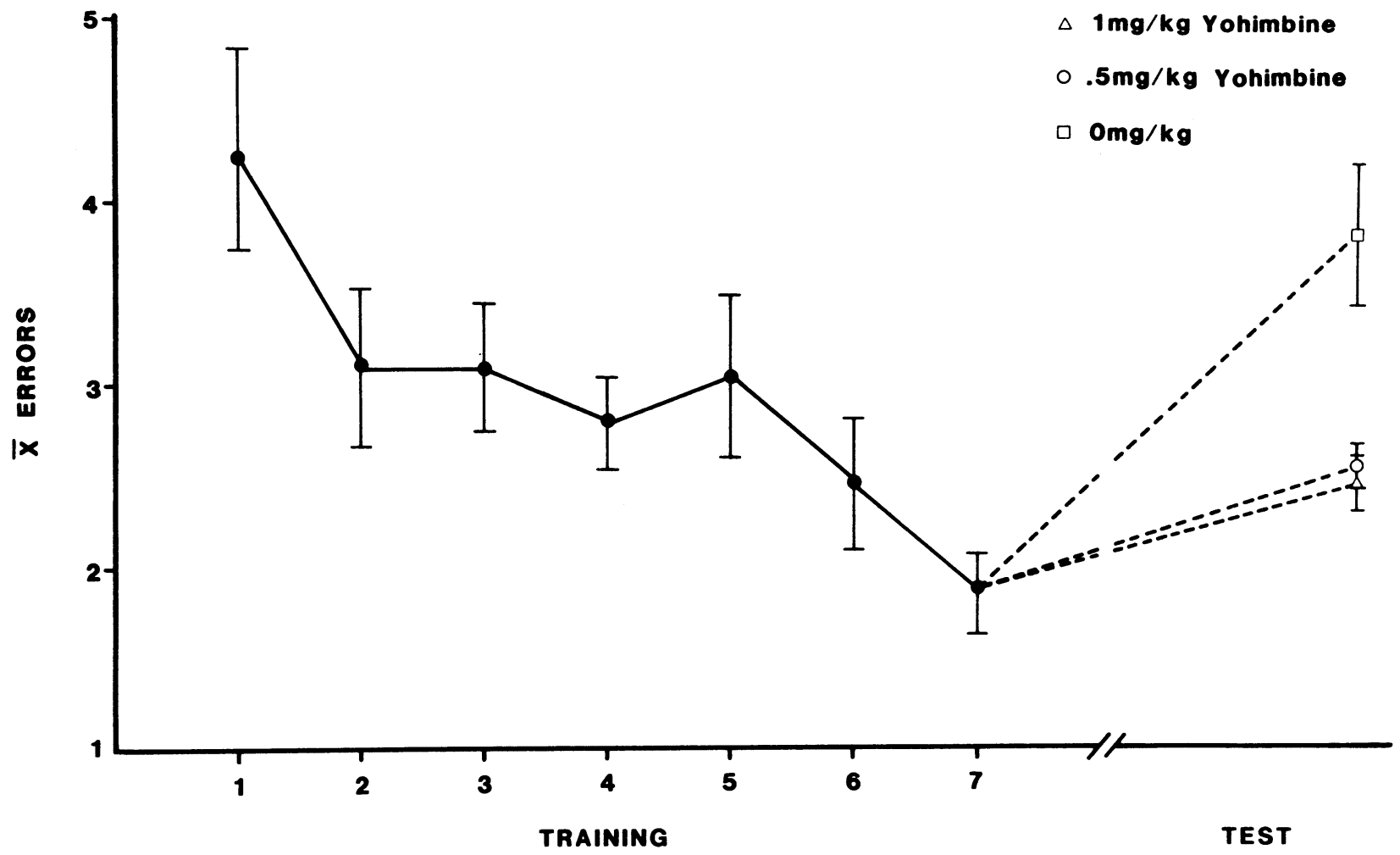

Figure 6. Mean number of errors at each trial during the 7 days of acquisition for the combined groups before treatment is shown at the left. Errors at retention test for each treatment group 25 days after the last training trial are shown at the right. Injections were given 25 min before the retention test. Note the forgetting in saline-injected control rats and the alleviation of forgetting by pretest treatment with yohimbine (from Sara, 1985).

Twenty rats were trained in the usual way, except that 25 min before the fourth daily training trial they were injected with saline or yohimbine $(0.5 \mathrm{mg} / \mathrm{kg}$ ip). The fourth trial was chosen because performance on this trial is roughly what might be expected at a retention test 25 days later. There was no effect of the drug on the day it was administered.

Much to our surprise, when the rats were given a fifth trial $24 \mathrm{~h}$ later, without any injection, the rats that had received yohimbine the preceding day made fewer errors than saline-injected rats (Figure 7).

The experiment was repeated with 15 rats per group. The animals were injected with saline on Days 1 and 2 of training. They were divided into matched groups according to time, errors, and retracings on Day 2 . The drug treatment was administered on Day 3. Again, there was no effect of the drug on performance of the partially learned task, as can be seen in Figure 8. However, the drug-induced facilitation is evident in the performance of the rats on Days 4 and 5. A repeated measures analysis of variance of the number of errors on Days 3, 4, and 5 reveals a significant treatment effect $[F(1,89)=4.13$, $\mathrm{p}=.048]$. The repetition factor was not significant, nor was the interaction. It is important to note that there were no injections on Days 4 and 5. We suggest that the improvement occurs because the rat, under the influence of yohimbine, attends to more relevant stimuli, both $\mathrm{S}+\mathrm{s}$ and
S-s, and thus learns more on Day 3. On the following day, this better learning to approach the $\mathrm{S}+$ and avoid the $\mathbf{S}-$ is expressed in a more accurate performance. As discussed above, we have recent evidence that rats rely heavily on very subtle intramaze cues in this task. The response-produced cues, which we had initially believed to be the major discriminative stimuli, are not sufficient for learning, at least not in fewer than seven trials (Sara \& Dekeyne, 1985).

Yohimbine increases heart rate and blood pressure, and this could result in an increase of oxygen supply to the brain and improved cerebral metabolism (Goldberg \& Robertson, 1983). Cognitive-enhancing effects of yohimbine could be due to this, rather than to any direct neuronal modulation by increased NE activity. Central administration of yohimbine would not necessarily resolve this question, since the LC, itself, is involved in the regulation of cerebral blood flow. Several drugs currently used to improve cognitive functioning in psychogeriatric patients have been thought to have their effect through the common mechanisms of improved cerebral metabolism. It has recently been shown that several of these drugs (vincamine, hydergine, and piracetam) increase the firing of LC neurons (Olpe, Jones, \& Steinmann, 1983). These investigators suggest that this common effect of the drugs might be partially responsible for the enhancement of cognitive abilities demonstrated by patients. 


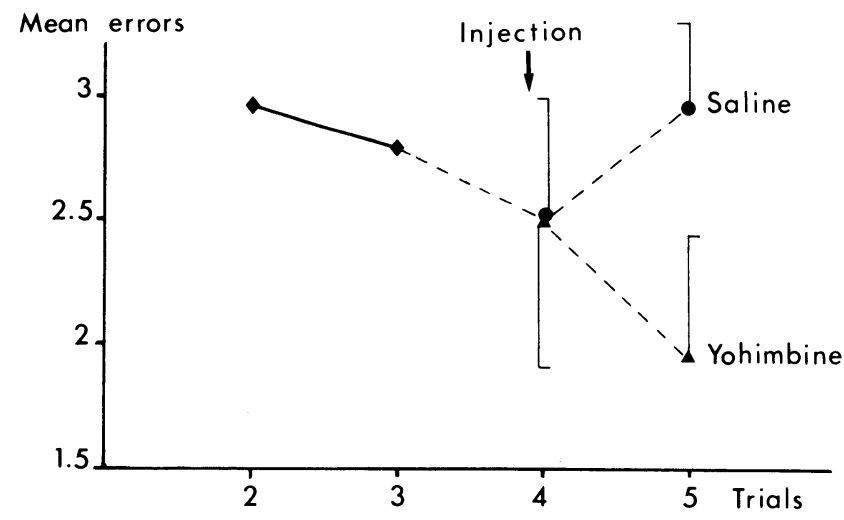

Figure 7. Mean errors during Trials 2 and 3 of acquisition for combined groups and at Trials 4 and 5 for saline- or yohimbine-treated $(.5 \mathrm{mg} / \mathrm{kg}$ ip) rats. Note that there was one trial per day and that the single injection occurred $25 \mathrm{~min}$ before Trial 4. There was no effect of the drug on this day; the effect was expressed in the facilitated performance of the yohimbinetreated group on Day 5, $24 \mathrm{~h}$ after yohimbine injection.

Nevertheless, whether or not the effect is mediated by direct modulatory action of NE on forebrain neurons remains an open question. Such a question must be addressed by a combined behavioral, pharmacological, and electrophysiological approach.

\section{LOCUS COERULEUS ACTIVITY AND ATTENTION-ELICITING SITUATIONS}

If $\mathrm{NE}$ does play such a role in modulating forebrain responsiveness as a function of the significance of the signal, then a key to understanding the regulation of attentional processes lies in the regulation of LC activity and reactivity. LC should increase its firing in situations that require maximal attention to the environment and should

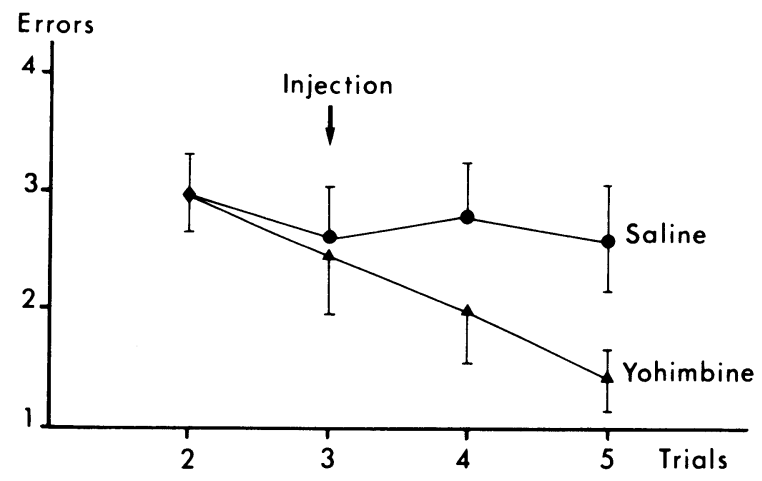

Figure 8. Mean errors for the combined groups at the second acquisition trial and for the saline- or yohimbine-treated groups on Trials 3, 4, and 5 . All rats were injected with saline before daily Trials 1 and 2 . They were treated with saline or yohimbine $(.5 \mathrm{mg} / \mathrm{kg}$ ip) before Trial 3 and received no injection before Trials 4 and 5 . Note that, as in the previous experiment, there was no effect of the drug on performance on the day it was injected. Facilitation of learning is expressed in behavior on the following day, on which no drug was administered. not fire when this attention might interfere with on-going homeostatic-directed behavior. And, indeed, Aston-Jones and Bloom (1981) have shown just that. The LC is inhibited during paradoxical sleep and during grooming and consummatory behavior. It produces its highest rate of firing when the organism orients to a novel stimulus. It remains to be demonstrated that the same neurons would fire to stimuli that have an acquired biological significance (conditioned stimuli).

Experiments showing the dependence of retrieval of weak memories on the context or background have led us to propose that contextual cue reminders elicit an LC response, which in turn facilitates attention to, or discrimination of, the CS. Thus, the LC should respond preferentially to the context, rather than to the discriminative stimulus. This preparatory response, which releases NE in the forebrain should result in improved stimulus selection and discrimination.

In an attempt to test this hypothesis, we were able to measure an increase in hippocampal MHPG, the major metabolite of NE, in response to an exposure to the context in which a rat had received a mild footshock $24 \mathrm{~h}$ previously (Sara, Guibert, \& Leviel, 1985). It is not clear from these experiments, however, whether the increased $\mathrm{NE}$ activity was due to a conditioned LC response to the context or was a residual long-term effect of the footshock, itself, on LC activity. Another way to approach this question would be to record LC activity in rats during exposure to context and/or the conditioned stimulus, as has been done during the orienting response (Aston-Jones \& Bloom, 1981). An electrophysiological measure is apparently more sensitive to small changes in cell firing than is a biochemical measure of transmitter release in a target structure. Indeed, it appears that increases of up to $30 \%$ in LC firing cannot be detected by changes in forebrain neurochemistry (H. R. Olpe, personal communication, 1985). 


\section{CONCLUSIONS}

Our experiments have shown that lesions to the coeruleocortical system do not impair cognitive behavior, even when there is an undetectable level of NE in the cortex or the hippocampus. Rats with such lesions are able to acquire a complex discrimination task, they have normal long-term retention and retrieval abilities, and they are no worse at reversal than normal rats. Furthermore, the paradigms that reveal this lack of effect of NE depletion are the same paradigms that have been used to show clear-cut improvement of learning and memory when NE is pharmacologically stimulated, so it is not the behavioral measure which is insensitive to NE manipulation. Moreover, those animals which do not show a recovery of NE, either through direct measure of forebrain content or implicitly through a normal PDR, perform significantly better than normal rats and rats that show this recovery.

The lack of any robust cognitive deficit in the face of persistent NE depletion clearly suggests a functional recovery, perhaps one involving a restoration of oxidative metabolism and/or Na-K-ATPase activity, as discussed above. The better performance of the nonrecovered rats suggests that there might be a functional overshoot. Perhaps, in some cases, the system is only partially destroyed, with a resultant increase in the firing of LC neurons (Chiodo et al., 1983). This would be functionally equivalent to a stimulation of the system by yohimbine, thus accounting for the observed facilitation in these animals.

Yohimbine produces clear-cut facilitatory effects in memory retrieval paradigms, which, we have argued, make particular demands on selective attention (Sara, 1985). Long-term depletion of forebrain NE does not produce behavioral deficits in the same paradigms. The facilitation of these cognitive processes by yohimbine, a drug which increases LC firing, argues in support of the hypothesis that the coeruleocortical NE system is involved in such processes. On the other hand, because of the demonstrated rapid recovery of NE function, the lack of impairment after lesions to the system would not be considered as strong evidence against a role for this system in attention, learning, and memory processes.

These results suggest that the lesion technique is not an appropriate way to measure function, at least with respect to the coeruleocortical system. The pharmacological approach is promising, but it, too, has its limitations. As discussed above, stimulation or impairment of function of LC might have an effect on peripheral factors such as blood pressure or rate of cerebral blood flow, which could account for any cognitive impairment or enhancement, even with intracranial injections. Iontophoretic application of NE to target structures in appropriate behavioral situations, although technically difficult (but see West \& Woodward, 1984), will probably prove to be the best method with which to test the hypothesis that the ob- served NE modulation of signal/noise in the forebrain is related to selective attention, learning, and memory.

Meanwhile, recording of neuronal activity in the LC of the rat in the process of selecting, attending, and remembering is possible, and these kinds of experiments should furnish important data concerning the conditions under which the LC is activated. Amaral and Sinnamon (1977) proposed that the LC, as the central analogue of a sympathetic ganglion, "serves to maintain levels of excitability optimal for information processing of environmental stimuli relevant to coping with an aversive event" (p. 184). In our view, the LC will fire not only during situations of stress or novelty, but also in response to rather specific stimuli which have a biological significance by virtue of their previous association with reinforcement. Thus, the LC might be considered as a kind of divergent interface, transforming this relatively specific information concerning, for example, significance of a context, into a general arousal, so as to increase the processing capacity of target forebrain structures. The modulatory effect on target structures, of the conditioned response of LC to context, prepares the hungry rat for the cognitive integration (stimulus selection, response organization, etc.) required for adaptive behavior, such as negotiating a maze to find food.

\section{REFERENCES}

Adrien, J., Buisseret, P., Frégnac, Y., Gary-Bobo, D., Imbert, M., TAssin, J. P., \& TrotTer, Y. (1982). Noradrénaline et plasticité du cortex visuel du chaton: Un réexamen. Comptes-Rendus de l'Académie des Sciences (Paris), 295, 745-750.

Amaral, P. G., \& Sinnamon, H. M. (1977). The locus coeruleus: Neurobiology of a central noradrenergic nucleus. Progress in Neurobiology, 1, 147-196.

ArChER, T., Cotic, T., \& JÄrbe, T. U. (1982). Attenuation of the context effect and lack of unconditioned stimulus-preexposure effect in taste-aversion learning following treatment with DSP4, the selective noradrenaline neurotoxin. Behavioral \& Neural Biology, 35, 159-173.

ArCher, T., Mohammed, A. K., \& JÄrBE, T. U. (1983). Latent inhibition following systemic DSP4: Effects due to presence and absence of contextual cues in taste aversion learning. Behavioral \& Neural Biology, 38, 287-306.

Aston-Jones, G. (1985). Behavioral functions of locus coeruleus derived from cellular attributes. Physiological Psychology, 13, 118-126.

Aston-Jones, G., \& BLOOM, F. (1981). Norepinephrine-containing locus coeruleus neurons in behaving rats exhibit pronounced responses to non noxious environmental stimuli. Journal of Neuroscience, 1 , $887-900$.

Chiodo, A., Acheson, A., Zigmond, J., \& Stricker, M. (1983). Subtotal destruction of central noradrenergic projections increases the firing rate of locus coeruleus cells. Brain Research, 264, 123-126.

Crow, T. J., Deakin, J., File, S. E., Longden, A., \& Wendlandt, S. (1978). The locus coeruleus noradrenergic system: Evidence against a role in attention, habituation, anxiety, and motor activity. Brain Research, 155, 249-261.

Daw, N. W., Rader, R., Robertson, T., \& Videen, T. (1983). Do short term and long term depletion of noradrenaline have different effects on visual deprivation in the latter visual cortex. Journal of Neuroscience, 4, 1354-1360.

Descarries, L., Watkins, K., \& LapierRe, Y. (1977). Noradrenergic axon terminals in the cerebral cortex of rat. III. Topometric ultrastructural analysis. Brain Research, 133, 197-222. 
DeweEr, B., \& SARA, S. J. (1984). Background stimuli as a reminder after spontaneous forgetting: Role of duration of cuing and cuing-test interval. Animal Learning \& Behavior, 12, 238-247.

Deweer, B., Sara, S. J., \& Hars, B. (1980). Contextual cues and memory retrieval in rats: Alleviation of forgetting by a pretest exposure to background stimuli. Animal Learning \& Behavior, 8, 265-272.

Dismukes, E. (1977). New look at the aminergic nervous system. $\mathrm{Na}$ ture, 269, 557-558.

Gervais, R., \& PAGER, J. (1983). Olfactory bulb excitability selectively modified in behaving rats after local 6-hydroxydopamine treatment. Behavioural Brain Research, 9, 165-179.

Gisquet-Verrier, P., Dekeyne, A., \& Alexinsky, T. (1985). Memory reorganization over time as revealed by interaction between type of pre-test cueing and length of retention interval. In B. Will, P. Schmitt, \& R. Dalrymple-Alford (Eds.), Brain plasticity, learning and memory. New York: Plenum Press.

GoldBerg, M., \& RoberTson, D. (1983). Yohimbine: A pharmacological probe for study of the alpha2-adrenoreceptor. Pharmacological Reviews, 35, 143-180.

Guibert, A., SARA, S. J., \& Leviel, V. (1983). [Correlation between cortical and hippocampal levels of noradrenaline and latency and duration of post decapitation clonus after treatment with DSP4.] Unpublished manuscript, CNRS, Gif-sur-Yvette, France.

Hallman, H., SundsTröm, D., \& Jonsson, G. (1984). Effects of the noradrenaline neurotoxin DSP4 on monoamine neurons and their transmitter turnover in rat CNS. Journal of Neural Transmission, 60, 89-102.

HARIK, S. (1984). Locus coeruleus lesion by local 6-hydroxydopamine infusion causes marked and specific destruction of noradrenergic neurons, long-term depletion of neuropinephrine and the enzymes that synthetize it, and enhanced dopaminergic mechanisms in the ipsilateral cortex. Journal of Neuroscience, 4, 699-707.

Harik, I., Duckrow, R., La Manna, C., Rosenthal, M., Sharma, K., \& BANERJEE, P. (1981). Cerebral compensation for chronic noradrenergic denervation induced by locus coenuleus lesion: Recovery of receptor binding, isoproterenol-induced adenylate cyclase activity and oxidative metabolism. Journal of Neuroscience, 6, 641-649.

Harrell, L., Barlow, T., Miller, M., Haring, J., \& Davis, J. (1984). Facilitated reversal learning of a spatial memory task by medial septal injection of 6-hydroxydopamine. Experimental Neurology, 85, 69-77.

Hobson, J. (1980). Toward a cellular neurophysiology of the reticular formation: Conceptual and methodological milestones. In J. Hobson \& M. Brazier (Eds.), The reticular formation revisited. New York: Raven Press.

Kasamatsu, T., \& Pettigrew, J. (1976). Depletion of brain catecholamines: Failure of ocular dominance shift after monocular occlusion in kittens. Science, 194, 206-209.

Kasamatsu, T., Pettigrew, J., \& Ary, M. (1979). Restoration of visual cortical plasticity by local microperfusion of norepinephrine. Journal of Comparative Neurology, 185, 163-182.

Kasamatsu, T., \& Shirokawa, T. (1985). Are beta adrenoreceptors involved in visuocortical plasticity? In B. Will, P. Schmitt, \& R. Dalrymple-Alford (Eds.), Brain plasticity, learning and memory. New York: Plenum Press.

KeTY, S. (1970). The biogenic amines in the central nervous system: Their possible roles in arousal emotion and learning. In F. O. Schmitt (Ed.), The neurosciences (pp. 324-336). New York: Rockefeller University Press.

KonorsKI, J. (1967). Integrative activity of the brain. Chicago: Chicago University Press.

KuPalov, P. (1961). Some normal and pathological processes in the brain. In N. Kline (Ed.), Pavlovian Conference on Higher Nervous Activity. Annals of the New York Academy of Sciences, 92, 1046-1053

La Manna, J., Harik S., Light, A., \& Rosenthal, M. (1981). Norepinephrine depletion alters cerebral oxidative metabolism in the "active" state. Brain Research, 204, 87-101.
Lorden, J. F., Rickert, E., Dawson, R., \& Pellymounter, M. (1980). Forebrain norepinephrine and the selective processing of information. Brain Research, 190, 569-573.

MADAR, Y., \& SEGAL, M. (1980). The functional role of the noradrenergic system in the visual cortex: Activation of the noradrenergic pathway. Experimental Brain Research, 41, 814.

Mason, S., \& Iversen, S. (1978). Reward, attention and the dorsal noradrenergic bundle. Brain Research, 150, 135-148.

Moore, R. Y. (1980). The reticular formation: Monoamine neuron system. In J. A. Hobson \& M. A. Brazier (Eds.), The reticular formation revisited (pp. 67-81). New York: Raven Press.

Olpe, H. R., Jones, R., \& Steinmann, M. (1983). The locus coeruleus: Actions of psychoactive drugs. Experientia, 39, 242-249.

Olschowka, J., Molliver, M., Grzanna, R., Rice, F., \& Coyle, J. (1981). Ultrastructural demonstration of noradrenergic synapses in the rat central nervous system by dopamine-B-hydroxylase immunocytochemistry. Journal of Histochemistry \& Cytochemistry, 29, 271-280.

PICKLES, J. O. (1976). The noradrenaline-containing innervation of the cochlear nucleus and the detection of signals in noise. Brain Research, 105, 591-596.

PisA, M., \& Fibiger, H. C. (1983). Evidence against a role of the rat's dorsal noradrenergic bundle in selective attention and place memory. Brain Research, 272, 319-329.

Quartermain, D. (1983). The role of catecholamines in memory processing. In J. A. Deutsch (Ed.), The physiological basis of memory (2nd ed). New York: Wiley.

READER, T. (1983). The role of catecholamines in neuronal excitability. In A. R. Liss (Ed.), Basic mechanisms of neuronal hyperexcitability (pp. 281-321). New York: Liss.

RoBBins, T. W. (1984). Cortical noradrenaline, attention and arousal. Psychological Medicine, 14, 13-21.

Robbins, T. W., \& Everitt, H. B. (1985). Noradrenaline and selective attention. In B. Will, P. Schmitt \& R. Dalrymple-Alford (Eds.), Brain plasticity, learning and memory, New York: Plenum Press.

Ross, S. (1976). Long-term effects of N-2-chloroethyl-N-ethyl-2bromobenzylamine hydrochloride on noradrenergic neurons in the rat brain and heart. British Journal of Pharmacology, 58, 521-527.

SARA, S. J. (1985). Noradrenergic modulation of selective attention: Its role in memory retrieval. In D. Olton, E. Gamzu, \& S. Corkin (Eds.), Memory dysfunctions: An integration of animal and human research from clinical and preclinical perspectives. Annals of the New York Academy of Sciences, 444, 178-193.

SARA, S. J., \& DEKEYNE, A. (1985). [Role of intramaze visual cues in performance of a "spatial" discrimination multi unit maze task.] Unpublished manuscript, CNRS, Gif-sur-Yvette, France.

SARA, S. J., \& DEWEER, B. (1982). Memory retrieval enhanced by amphetamine after a long retention interval. Behavioral \& Neural Biology, 26, 146-160.

Sara, S. J., Deweer, B., \& Hars, B. (1976). Forgetting as a lapse not a loss: Facilitation of retrieval by a reminder. Bulletin d'Information de la Société Belge de Psychologie, 1, 22-25.

Sara, S. J., Deweer, B., \& HaRS, B. (1980). Reticular stimulation facilitates retrieval of a "forgotten" maze habit. Neurosciences Letters, 18, 211-217.

Sara. S. J., Guibert, B., \& Leviel, V. (1985). Conditioned increase in hippocampal MHPG in response to contextual stimuli associated with a brief, mild, footshock. In Fifth European Winter Conference on Brain Research. (Abstract)

SARA, S. J., \& LERoch, K. (1985) [Yohimbine effects on spontaneous locomotor activity, exploration and neophobia as measured in the roleboard: A dose-response study.] Unpublished manuscript, CNRS, Gifsur-Yvette, France.

SEGAL, M. (1981). The action of norepinephrine in the rat hippocampus: Intracellular studies in the slice preparation. Brain Research, 206, 107-128.

SEGAL, M. (1982). The action of serotonin in the rat hippocampus. In B. Haber (Ed.), Serotonin-current aspects of neurochemistry and function. New York: Plenum Press. 
SEgAL, M. (1985). Mechanisms of action of noradrenaline in the brain. In B. Will, P. Schmitt, \& R. Dalrymple-Alford (Eds.), Brain plasticity, learning and memory. New York: Plenum.

Segal, M., \& Bloom, F. (1976). Norepinephrine in the rat hippocampus. III. Stimulation of nucleus locus coeruleus in the awake rat. Brain Research, 107, 499-511.

SEgal, M., \& Edelson, A. (1978). Effects of priming stimulation of catecholamine containing nuclei in rat brain on runway performance. Brain Research Bulletin, 3, 203-206.

Segal, M., Sagie, B., \& Mayevsky, A. (1980). Metabolic changes induced in rat hippocampal slices by norepinephrine. Brain Research, 202, 387-399.

SwanN, C. (1984). Brain $\left(\mathrm{Na}^{+}, \mathrm{K}^{+}\right)$-ATPase and noradrenergic function: Recovery of enzyme activity after norepinephrine depletion. Brain Research, 321, 323-326.
Waterhouse, B. D., Moises, H., \& WoodWard, D. (1980). Locus coeruleus stimulation potentiates somatosensory cortical neuronal responses to different synaptic inputs. Society of Neuroscience Abstracts, 6, 448 .

Woodward, D. J., Moises, H. C., Waterhouse, B., Hoffer, B., \& FREedman, R. (1979). Modulatory actions of norepinephrine in the central nervous system. Federation Proceedings, 38, 2109-2116.

WEST, M. O., \& WoodWARD, D. J. (1984). A technique for microiontophoretic study of single neurons in the freely moving rat. Journal of Neuroscience Methods, 11, 179-186.

(Manuscript received July 8, 1985; revision accepted for publication October 20, 1985.) 